\section{Development of a multi-channel NIRS-USG hybrid imaging system for detecting prostate cancer and improving the accuracy of imaging-based diagnosis: a phantom study}

Heejin Bae', Seung-seob Kim', Seungsoo Lee ${ }^{1}$, Hyuna Song ${ }^{2}$, Songhyun Lee ${ }^{3}$, Dalkwon Koh², Jae Gwan Kim³ , Dae Chul Jung ${ }^{1}$

\begin{abstract}
'Department of Radiology, Research Institute of Radiological Science, Yonsei University College of Medicine, Seoul; ${ }^{2}$ Department of Biomicrosystem Engineering, Korea University College of Health Science, Seoul; ${ }^{3}$ Department of Medical System Engineering, School of Information and Communications, Gwangju Institute of Science and Technology, Gwangju, Korea
\end{abstract}

Purpose: This study aimed to develop a multi-channel near-infrared spectroscopy (NIRS) and ultrasonography (USG) fusion imaging system for imaging prostate cancer and to verify its diagnostic capability by applying the hybrid imaging system to a prostate cancer phantom.

Methods: A multi-channel NIRS system using the near-infrared 785-nm wavelength with 12 channels and four detectors was developed. After arranging the optical fibers around a USG transducer, we performed NIRS imaging and grayscale USG imaging simultaneously. Fusion imaging was obtained by processing incoming signals and the spatial reconstruction of NIRS, which corresponded with grayscale USG acquired at the same time. The NIRS-USG hybrid system was applied to a silicone-based optical phantom of the prostate gland containing prostate cancer to verify its diagnostic capability qualitatively.

Results: The NIRS-USG hybrid imaging system for prostate cancer imaging simultaneously provided anatomical and optical information with 2-dimensional registration. The hybrid imaging system showed more NIR attenuation over the prostate cancer model than over the model of normal prostate tissue. Its diagnostic capability to discriminate a focal area mimicking the optical properties of prostate cancer from the surrounding background mimicking the optical properties of normal prostate tissue was verified by applying the hybrid system to a silicone-based optical phantom of prostate cancer.

Conclusion: This study successfully demonstrated that the NIRS-USG hybrid system may serve as a new imaging method for improving the diagnostic accuracy of prostate cancer, with potential utility for future clinical applications.

Keywords: Prostate neoplasms; Spectroscopy, near-infrared; Ultrasonography; Hybrid probe; Fusion imaging

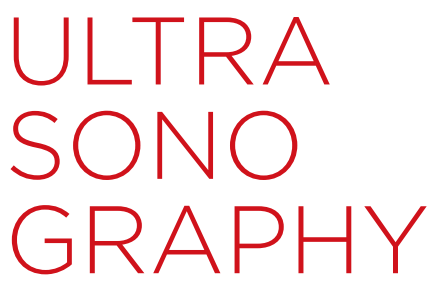

ORIGINAL ARTICLE

https://doi.org/10.14366/usg. 18030 pISSN: 2288-5919 • elSSN: 2288-5943 Ultrasonography 2019;38:143-148

Received: May 23, 2018 Revised: July 4, 2018 Accepted: July 14, 2018

Correspondence to: Dae Chul Jung, MD, Department of Radiology, Yonsei University College of Medicine, 50-1 Yonsei-ro, Seodaemungu, Seoul 03722, Korea

Tel. $+82-2-2228-7400$

Fax. +82-2-393-3035

E-mail: DAECHUL@yuhs.ac

This is an Open Access article distributed under the terms of the Creative Commons Attribution NonCommercial License (http://creativecommons.org/ licenses/by-nc/4.0/) which permits unrestricted noncommercial use, distribution, and reproduction in any medium, provided the original work is properly cited.

Copyright (C) 2019 Korean Society of Ultrasound in Medicine (KSUM)

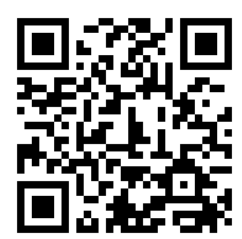

How to cite this article:

Bae $H$, Kim SS, Lee $S$, Song $H$, Lee $S$, Koh $D_{\text {, }}$ et al. Development of a multi-channel NIRSUSG hybrid imaging system for detecting prostate cancer and improving the accuracy of imaging-based diagnosis: a phantom study. Ultrasonography. 2019 Apr;38(2):143-148. 


\section{Introduction}

Prostate cancer is the second most frequently diagnosed cancer in the male population and the fifth leading cause of cancer deaths worldwide $[1,2]$. Transrectal ultrasound (TRUS)-guided prostate biopsy is the standard and most common method for detecting prostate cancer in men with elevated serum prostatespecific antigen levels and/or abnormal digital rectal examination findings [3]. Unlike many other solid tumors, for which imageguided targeted biopsy is common, prostate cancer is detected by random sampling of the entire prostate gland because imagingbased identification of prostate cancer is difficult. Consequently, the low cancer detection rate $(27 \%-40 \%)$ of TRUS-guided systematic random biopsies results in missing a substantial proportion of cases of clinically significant prostate cancer $[4,5]$.

Near-infrared spectroscopy (NIRS) is an optical method of measuring changes in the concentration of chromophores, using light with wavelengths in the range of $700-900 \mathrm{~nm}$, which is known to move by scattering rather than absorption, enabling it to reach deep inside the human body [6]. Since the chromophores in the human body consist mostly of oxyhemoglobin and deoxyhemoglobin, NIRS signals can reflect the status of biologic metabolism inside the human body [7]. In general, malignant tissue can be either hypervascular from overstimulated angiogenesis or hypovascular due to rapid tissue growth exceeding its vascular supply. Prostate cancer is known to show a higher concentration of microvasculature than normal prostate tissue [8]. Jiang et al. [9,10] demonstrated that NIRS could detect this hemodynamic difference between normal prostate tissue and prostate cancer, meaning that NIRS could be used to image prostate cancer with higher sensitivity and specificity than TRUS. According to their results, however, TRUS was more accurate in localizing prostate cancer than NIRS, especially in the deep portion of the prostate gland. Therefore, it might be possible to improve diagnostic accuracy by combining these two modalities. The purpose of this study was to develop an NIRS-ultrasonography (USG) hybrid imaging system and to verify its diagnostic capability using an optical phantom model of a normal prostate gland and prostate cancer.

\section{Materials and Methods}

Institutional review board at our institution approved this study, and the requirement for informed consent was waived.

\section{Development of the Multi-channel NIRS-USG Hybrid Imaging System}

The continuous wave method was used to measure NIRS signals in our system, with a wavelength of $785 \mathrm{~nm}$. A total of 12 laser diodes (Thorlabs, Newton, NJ, USA) were used as the light source in the multi-channel NIRS system. A total of four avalanche photodiodes (APDs) (C5460-01, Hamamatsu Photonics, Hamamatsu, Japan) were used as detectors to improve the spatial resolution and the field of view. After passing the band pass filter, light was demodulated into its direct current component by the demodulation circuit using a root mean square detector. The signal lost during this demodulation process was compensated by a signal amplifier using OP-Amp. It then underwent a low-pass filter to remove the remaining modulated component. Finally, it was sampled by a data acquisition device (PCI-6122, National Instruments, Austin, TX, USA), through two channels simultaneously. To determine the switching and sampling rates of the laser diodes, a microcontroller unit (MCU) (ATmega128) was used. The maximal sampling rate was set at 30 $\mathrm{Hz}$ by the MCU. Optical fibers with a diameter of $400 \mu \mathrm{m}$ (FT400EMT, Thorlabs) were connected to the laser diodes and APDs. The intensity of the emitted laser was set between 5 and $7 \mathrm{~mW}$. Labview software (National Instruments) was used to develop a graphical user interface that could process the incoming signals and display them on the same monitor simultaneously. The open-source platform Near-Infrared Fluorescence and Spectral Tomography (NIRFAST; Dartmouth College and University of Birmingham, Birmingham, UK), which simulates light propagation in biological tissue, was used for modeling near-infrared (NIR) light transport inside the phantom $[11,12]$. This software, coded on the basis of MATLAB (MathWorks, Natick, MA, USA), is capable of retrieving the absorption coefficient from single-wavelength light attenuation and reconstructing it into 2-dimensional (2D) images.

A plastic holder for a high-frequency linear ultrasound (US) transducer (12-15 MHz) of an Aixplorer US system (Super-Sonic Imagine, Aix-en-Provence, France) was also developed. It contained 12 holes with the same diameter as the optical fibers along two facing long sides with a rectangular shape. These holes were designed to hold the optical fibers firmly, preventing them from moving during the signal. The arrangement of the sources and detectors was also changed to be symmetrical on both sides of the plastic holder to minimize the interference caused by nearby sources. The hybrid imaging systems were completed by assembling a US probe holder, a controllable metal arm with two joints, and a platform for a phantom (Fig. 1A, B).

\section{Development of the Optical Phantom Containing a Prostate Cancer Model}

A phantom with optical properties mimicking those of a prostate gland with prostate cancer inside was developed using $\mathrm{TiO}_{2}$ for the scattering effect and India ink for the absorption effect [13]. 
Reference values of the optical properties of normal prostate tissue, prostate cancer, and the surrounding muscle layers were selected from previous studies [14-18] and expressed by adjusting the quantity of $\mathrm{TiO}_{2}$ and India ink. Following the fabrication process, a small phantom with optical properties mimicking the prostate cancer was made. Then, a larger phantom with optical properties mimicking those of a normal prostate gland was developed. During the process of making this standard prostate gland phantom, the prostate cancer phantom was placed into the mixed silicone before it was poured into a mold. Similarly, the larger phantom with optical properties similar to those of the muscle layer was made by placing the prostate gland phantom that contained the prostate cancer phantom into the muscle layer phantom before pouring the mixed silicone into a mold. Diagrams of the optical phantoms of the prostate gland containing prostate cancer are shown in Fig. 1C. A detailed specification of the materials used in phantom development is presented in Table 1.

\section{Verification of the Hybrid Imaging System Using the Optical Phantom Model of Prostate Cancer \\ The US transducer was placed within a plastic holder containing a total of 12 optical fibers (8 sources and 4 detectors) over the}

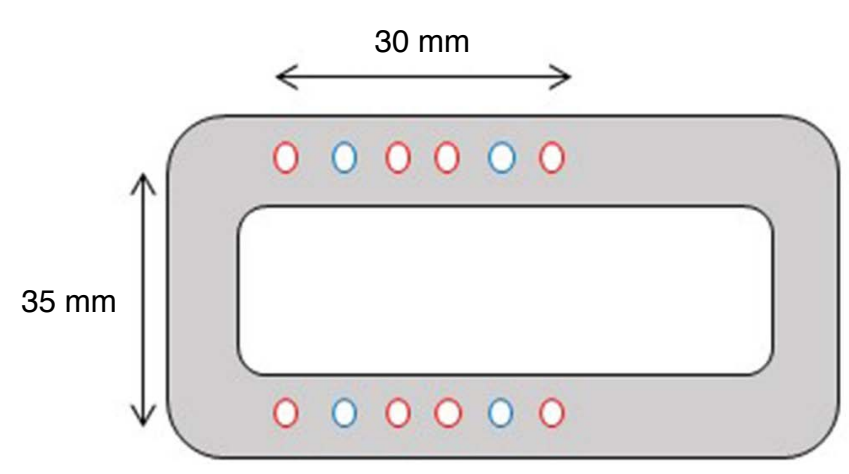

A

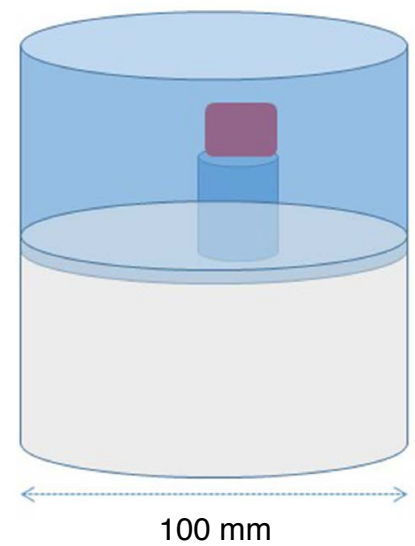

C

Fig. 1. A near-infrared spectroscopy and ultrasonography hybrid imaging system and the optical phantom of the prostate gland with the prostate cancer embedded inside.

A. Diagram of the plastic holder containing 12 holes is shown. The red holes contained source fibers with a wavelength of $785 \mathrm{~nm}$, while the blue holes were for detector fibers. B. The plastic holder with 12 holes, each containing optical fibers is placed on the phantom. C. Developed optical phantom of a normal prostate gland with prostate cancer is depicted in this diagram. Blue, gray, and red colors represent the prostate gland, muscle layer, and prostate cancer, respectively. The red-colored prostate cancer phantom is embedded inside the optical phantom of a normal prostate gland. D. The plastic holder with an ultrasound transducer is placed over the phantom. 
Table 1. Detailed specifications of the developed optical phantoms

\begin{tabular}{lcccccc}
\hline & Size $\left.(\mathrm{cm})^{\mathrm{a}}\right)$ & Amount $(\mathrm{mL})^{\mathrm{b})}$ & $\mathrm{TiO}_{2}(\mathrm{~g})$ & India ink $(\mathrm{g})$ & $\mu_{\mathrm{s}}{ }^{\prime}(/ \mathrm{cm}, 785 \mathrm{~nm})$ & $\mu_{\mathrm{a}}(/ \mathrm{cm}, 785 \mathrm{~nm})$ \\
\hline Prostate cancer & $1.2(\mathrm{w}), 1.4(\mathrm{l}), 0.8(\mathrm{~h})$ & 100 & 0.084 & 0.054 & 7.1 & 0.41 \\
Prostate gland & $4.3(\mathrm{w}), 4.5(\mathrm{l}), 5.3(\mathrm{~h})$ & 200 & 0.252 & 0.064 & 10 & 0.2 \\
Muscle layer & $13(\mathrm{ud}), 10(\mathrm{ld}), 6(\mathrm{~h})$ & 275 & 0.1 & 0.045 & 3.06 & 0.12 \\
\hline
\end{tabular}

$\mathrm{TiO}_{2}$, titanium dioxide; $\mu_{s}^{\prime}$, induced scattering coefficient; $\mu_{\mathrm{a}}$, absorption coefficient; $w$, width; l, length; $h$, height; ud, diameter of the upper face; Id, diameter of the lower face. ${ }^{a}$ Final size of a cured phantom. ${ }^{\text {b) }}$ Amount of mixed silicone prepared.

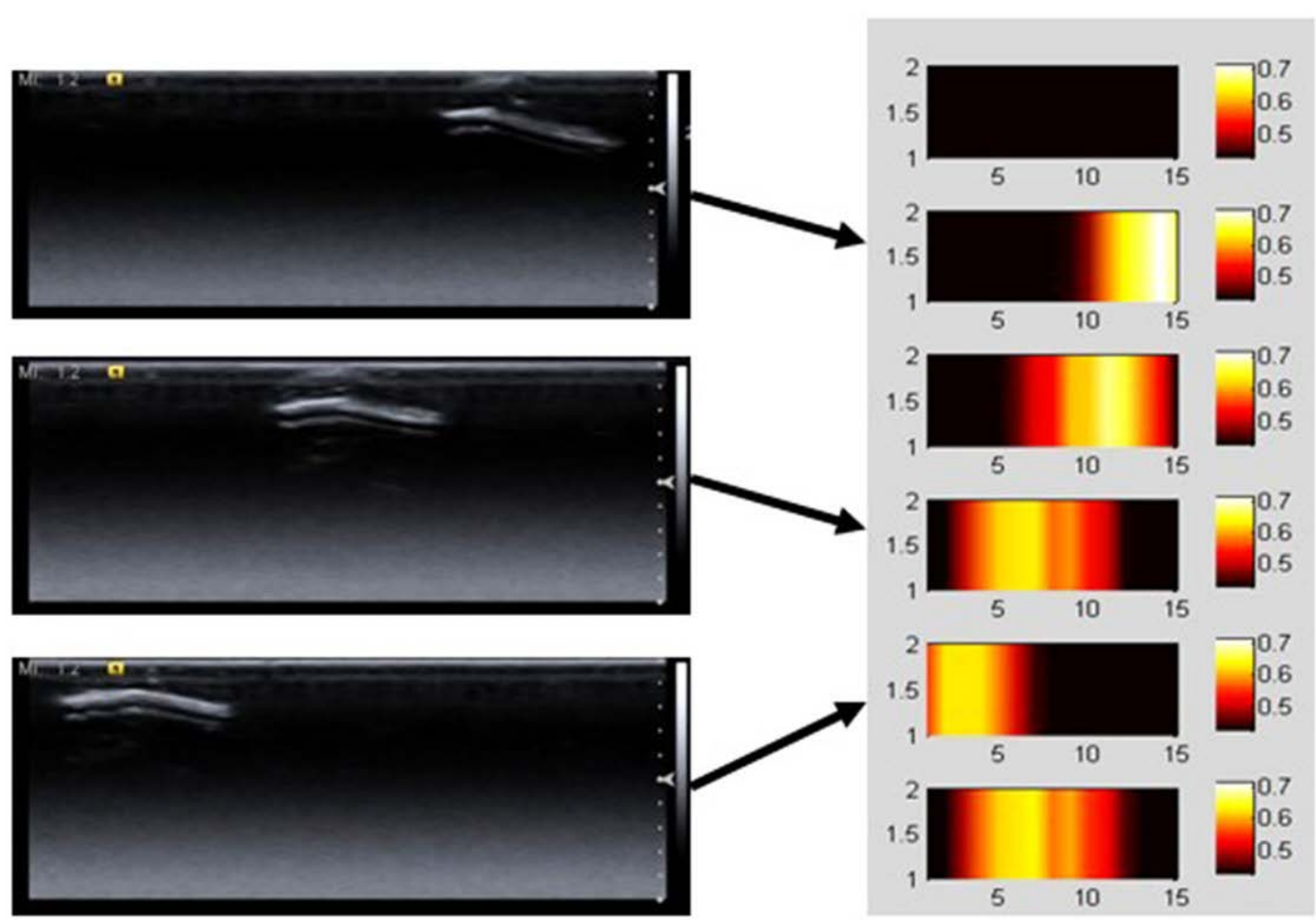

A
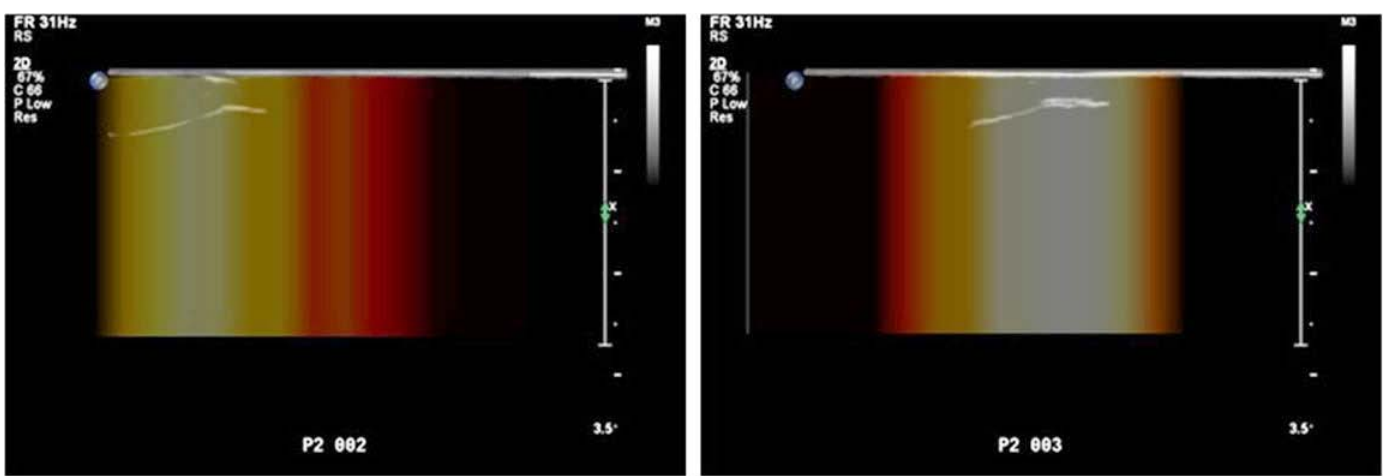

B

Fig. 2. Reconstructed 2-dimensional (2D) images, obtained by the near-infrared spectroscopy (NIRS) and ultrasonography (USG) hybrid imaging system using an optical prostate phantom.

A. The left column shows grayscale USG from three different locations of the hybrid probe placed over the prostate cancer. Arrows indicate the corresponding reconstructed 2D images from NIRS. The right column shows the reconstructed 2D images based on absorption coefficients. B. Overlaid images of the grayscale USG and the reconstructed 2D images are shown. Areas of high absorption coefficients were correlated with the location of the prostate cancer model. 
developed phantom, and NIRS signal attenuation and grayscale sonograms were obtained simultaneously (Fig. 1D). This hybrid imaging was repeated 4 times, and each time the location of the plastic holder over the prostate phantom was changed (Fig. 2). The collected NIRS signals were used to reconstruct the 2D images by modifying the NIRFAST software package, which simulates light propagation in biological tissue [11]. Fusion images of the reconstructed 2D images from the NIRS system and the crosssectional grayscale US images acquired at the same time were obtained.

\section{Results}

The intensity of the incoming NIR signals was relatively low when the hybrid probe was held over the prostate cancer phantom, suggesting that more light is absorbed and/or scattered when passing through these routes, reflecting the presence of an inner region with different optical properties from its surroundings under the location of the plastic holder. Rectangular 2D reconstruction images were formed iteratively, until the difference between the forward data and the reconstructed data did not improve by more than $2 \%$ when compared with the previous iteration. The final reconstructed $2 \mathrm{D}$ images based on the absorption coefficients of NIRS are shown in the right column of Fig. $2 \mathrm{~A}$ and overlaid images with grayscale USG are shown in Fig. 2B. As we changed the location of the optical fibers, the area of high absorption coefficients also changed, suggesting the capability of the NIRS system to reliably distinguish inner regions with different optical properties. The region with higher absorption coefficients was correlated with the location of the embedded optical phantom of prostate cancer. The longitudinal sonograms were simultaneously obtained during NIRS imaging, and the corresponding NIRS region is depicted with arrows in Fig. 2A. Three overlaid images of simultaneously obtained USG and the reconstructed $2 \mathrm{D}$ images are shown in Fig. 2B. Similarly, areas of high absorption coefficients were correlated with the location of the prostate cancer. The results show that good separation was obtained between the focal area mimicking the optical properties of prostate cancer from the surrounding background areas mimicking the optical properties of normal prostate tissue along the transverse axis. The hybrid imaging showed more NIR attenuation over the prostate cancer than over the normal prostate tissue, which may indicate its capability to discriminate focal areas mimicking prostate cancer from surrounding normal prostate tissue.

\section{Discussion}

In the present study, we developed a NIRS-USG fusion imaging system for prostate cancer imaging. It is non-invasive and simultaneously provides anatomical and optical (hemodynamic) information. Its diagnostic capability was qualitatively verified by applying the hybrid system to a silicone-based optical phantom of prostate cancer. The fusion imaging device was fabricated by arranging optical fibers around a US transducer. The hybrid imaging system showed more NIR attenuation over the prostate cancer model than over the normal prostate gland model. Because elevation of total hemoglobin levels is related to NIR attenuation, NIR imaging can detect tumors with increased vascularity, such as prostate cancer. Most of the optical contrast is probably due to intrinsic tumor vascularity and tumor-bound chromophores. Prostate cancer lesions are known to have a higher density of capillaries than the surrounding benign prostate tissue $[8,19]$. Jiang et al. $[9,10]$ demonstrated that NIRS could differentiate prostate cancer from the surrounding normal prostate gland using differences in optical properties caused by different degrees of vascularity, with better sensitivity and specificity than TRUS. It was anticipated that NIRS data would provide additional information about the localization of focal prostate cancer. This phantom study was an initial step in the development of a new NIRS imaging system and the verification of its diagnostic capabilities using an optical phantom of prostate cancer.

Kim et al. [18] previously developed an NIRS system used with an optical prostate phantom. In this study, the previous NIRS system and the fabrication process of optical phantom development were upgraded to optimize the system and to minimize the limitations of the previous study. First, there were several small air bubbles around the border between the normal prostate gland and muscle layer portions, which could have been a source of signal noise. A vacuum oven and a more powerful vacuum pump were used to minimize the air bubbles. Despite using an upgraded vacuum pump to remove the air bubbles generated along the interfaces between the two different materials during phantom fabrication, USG revealed that sheet-like air bubble layers remained along the interfaces between the normal prostate gland and the prostate cancer phantoms, which could have been the source of reverberation artifacts. Second, the optical fibers were not firmly fixed in position in the previous study, possibly reducing the reproducibility and reliability of the measurements. In the present study, we developed a plastic holder with metal arms to place each optical fiber in the same position during the measurements, and the received signals were remarkably more consistent. Finally, we adopted the NIRFAST algorithm to reconstruct 2D images, which became possible by adding more detectors to improve the spatial resolution and field of view. Therefore, the present hybrid system showed more prominent delineation of the focal area, with high absorption coefficients, from its surroundings, 
and the delineated focal area was closely correlated with the actual location of the target region.

Further studies will be performed to develop a clinically feasible hybrid imaging system. An animal study using a prostate cancer model should be performed to verify the in vivo diagnostic performance of this new imaging modality. As hybrid imaging can be performed on in vivo models, as well as artificially developed optical phantoms, an algorithm for image registration between NIRS and TRUS can be set up and optimized. Finally, a clinical study of prostate cancer patients should be conducted to validate and optimize the diagnostic performance of this modality on human bodies.

This study demonstrated that our NIRS-USG hybrid imaging system may serve as a new imaging method with potential for improving diagnostic accuracy in the localization of focal prostate cancer. In future, NIRS may be combined with TRUS, improving the diagnostic accuracy of prostate cancer imaging and the localization of target biopsy sites. This study presented a NIRS-USG hybrid imaging system for prostate cancer that was evaluated using a phantom, with potential utility for future clinical applications.

ORCID: Heejin Bae: https://orcid.org/0000-0002-1227-8646; Dae Chul Jung: https://orcid.org/0000-0001-5769-5083

\section{Conflict of Interest}

No potential conflict of interest relevant to this article was reported.

\section{Acknowledgments}

This work was supported by a National Research Foundation of Korea grant funded by the Korean Government (MEST) (NRF2016R1D1A1B03935144).

\section{References}

1. Torre LA, Bray F, Siegel RL, Ferlay J, Lortet-Tieulent J, Jemal A. Global cancer statistics, 2012. CA Cancer I Clin 2015;65:87-108.

2. Siegel RL, Miller KD, Jemal A. Cancer statistics, 2016. CA Cancer J Clin 2016;66:7-30.

3. Hodge KK, McNeal JE, Terris MK, Stamey TA. Random systematic versus directed ultrasound guided transrectal core biopsies of the prostate. J Urol 1989;142:71-74.

4. Djavan B, Ravery V, Zlotta A, Dobronski P, Dobrovits M, Fakhari M, et al. Prospective evaluation of prostate cancer detected on biopsies 1, 2, 3 and 4: when should we stop? J Urol 2001;166:1679-1683.

5. Guichard G, Larre S, Gallina A, Lazar A, Faucon H, Chemama S, et al. Extended 21-sample needle biopsy protocol for diagnosis of prostate cancer in 1000 consecutive patients. Eur Urol 2007:52:430-435.

6. Yodh A, Chance B. Spectroscopy and imaging with diffusing light.
Phys Today 1995;48:34-40.

7. Rolfe P. In vivo near-infrared spectroscopy. Annu Rev Biomed Eng 2000;2:715-754

8. Bigler SA, Deering RE, Brawer MK. Comparison of microscopic vascularity in benign and malignant prostate tissue. Hum Pathol 1993;24:220-226.

9. Jiang Z, Holyoak GR, Bartels KE, Ritchey JW, Xu G, Bunting CF, et al. In vivo trans-rectal ultrasound-coupled optical tomography of a transmissible venereal tumor model in the canine pelvic canal. J Biomed Opt 2009;14:030506.

10. Jiang Z, Piao D, Holyoak GR, Ritchey JW, Bartels KE, Slobodov G, et al. Trans-rectal ultrasound-coupled spectral optical tomography of total hemoglobin concentration enhances assessment of the laterality and progression of a transmissible venereal tumor in canine prostate. Urology 2011;77:237-242.

11. Dehghani H, Eames ME, Yalavarthy PK, Davis SC, Srinivasan S, Carpenter $\mathrm{CM}$, et al. Near infrared optical tomography using NIRFAST: algorithm for numerical model and image reconstruction. Commun Numer Methods Eng 2008:25:711-732.

12. Jermyn M, Ghadyani H, Mastanduno MA, Turner W, Davis SC, Dehghani $\mathrm{H}$, et al. Fast segmentation and high-quality threedimensional volume mesh creation from medical images for diffuse optical tomography. J Biomed Opt 2013;18:86007.

13. Ayers F, Grant A, Kuo D, Cuccia DJ, Durkin AJ. Fabrication and characterization of silicone-based tissue phantoms with tunable optical properties in the visible and near infrared domain. Proc SPIE Int Soc Opt Eng 2008;6870:687007.

14. Simpson CR, Kohl M, Essenpreis M, Cope M. Near-infrared optical properties of ex vivo human skin and subcutaneous tissues measured using the Monte Carlo inversion technique. Phys Med Biol 1998:43:2465-2478.

15. Svensson $T$, Andersson-Engels $S$, Einarsdottir $M$, Svanberg K. In vivo optical characterization of human prostate tissue using near-infrared time-resolved spectroscopy. J Biomed Opt 2007:12:014022.

16. Zhu TC, Dimofte A, Finlay JC, Stripp D, Busch T, Miles J, et al. Optical properties of human prostate at $732 \mathrm{~nm}$ measured in mediated photodynamic therapy. Photochem Photobiol 2005;81:96-105.

17. Sandell JL, Zhu TC. A review of in-vivo optical properties of human tissues and its impact on PDT. J Biophotonics 2011;4:773-787.

18. Kim SS, Jung DC, Song H, Lee S, Kim JG, Kim BM. Development of a multi-channel NIRS (near-infrared spectroscopy) imaging system for early detection and improvement of accuracy of imaging diagnosis in prostate cancer: preliminary phantom study. J Korean Soc Imaging Inform Med 2013;19:44-50.

19. Feng Y, Jeong EK, Mohs AM, Emerson L, Lu ZR. Characterization of tumor angiogenesis with dynamic contrast-enhanced MRI and biodegradable macromolecular contrast agents in mice. Magn Reson Med 2008;60:1347-1352. 